\title{
Controlled Growth of Non-Uniform Arsenic Profiles in Silicon Reduced-Pressure Chemical Vapor Deposition Epitaxial Layers
}

\author{
M. POPADIĆ, ${ }^{1,2}$ T.L.M. SCHOLTES,${ }^{1}$ W. DE BOER,${ }^{1}$ F. SARUBBI, ${ }^{1}$ \\ and L.K. NANVER ${ }^{1}$ \\ 1.-Laboratory of Electronic Components, Technology and Materials (ECTM), Delft Institute of \\ Microsystems and Submicrotechnology (DIMES), Delft University of Technology, Feldmannweg \\ 17, 2628 Delft, The Netherlands. 2.—e-mail: m.popadic@tudelft.nl
}

\begin{abstract}
An empirical model of As surface segregation during reduced-pressure chemical vapor deposition Si epitaxy is presented. This segregation mechanism determines the resulting doping profile in the grown layer and is here described by a model of simultaneous and independent As adsorption and segregation versus incorporation. The model quantifies this mechanism with enough detail to be successfully applied to the accurate growth of different profiles, including the ascending $x^{-2}$ doping profiles. For rapidly descending profiles the segregated As surface layer must be removed, e.g., by ex situ cleaning and Marangoni drying before further Si epitaxy.
\end{abstract}

Key words: Si epitaxy, reduced-pressure chemical vapor deposition (RPCVD), As doping, surface segregation, varactor diodes

\section{INTRODUCTION}

The ability to engineer abrupt, quickly varying, and unconventionally shaped doping profiles precisely can be used to improve the performance of Si-based devices. This has, in particular, been demonstrated for microwave and radio frequency $(\mathrm{RF})$ applications. ${ }^{1-4}$ However, it is often the doping fabrication technology that limits the range of devices that are feasible. Doping profiles of demanding shape cannot be achieved by ion implantation, and Si epitaxy remains the method of choice. Precise profile engineering demands slower growth rates, which, in the case of chemical vapor deposition (CVD), translates to low-temperature reduced-pressure chemical vapor deposition (RPCVD). However, in many situations, a bottleneck in the achievement of controllable As incorporation in low-temperature RPCVD Si epitaxy has been the strong surface segregation of this dopant. ${ }^{5}$

The effect of the surface segregation is to hamper any abrupt transition to another doping level, and,

(Received April 3, 2009; accepted July 9, 2009;

published online July 31,2009 ) in the case of an abrupt drop in doping, the undesired lingering of the higher As doping is referred to as autodoping. Previous experimental investigations have focused on exploring the optimal conditions for achieving goals such as suppressing lateral autodoping, ${ }^{6}$ determining the impact on the growth rate, ${ }^{7}$ growing highly-doped layers of poly-Si, ${ }^{8}$ or $\mathrm{Ge}_{x} \mathrm{Si}_{1-x}$ alloys. ${ }^{9}$ By the analysis of the mechanism governing the doping from the As segregated layer, good results were in the past achieved and applied to the growth of high-doped abrupt As peaks. ${ }^{10}$ However, none of the previous studies characterizes the growth parameters with enough detail to provide a general method for determining the growth parameters for non-uniform doping profiles.

In this paper we present an empirical model of As surface segregation that can be used for accurate growth of As profiles of demanding shapes. This model has been developed and successfully used for the realization of doping profiles that are perfectly proportional to the inverse square of the doping depth. Such profiles have been implemented in high-quality varactors that form the basis of circuit configurations that eliminate distortion. ${ }^{11,12}$ These circuits have demonstrated record-breaking 
linearity, ${ }^{13}$ enabled by the combination of novel design and the fact that the underlying doping profiles reach a sufficiently high degree of perfection. This paper gives the first presentation of the growth methodology used for these $x^{-2}$ profiles and also describes the method for other non-uniform profiles.

\section{EXPERIMENTAL PROCEDURES}

The starting material was 4 -in. $\langle 100\rangle n$-type $\mathrm{Si}$ wafers on which Si was epitaxially grown by CVD in the ASMI Epsilon One epitaxial reactor. The growth was performed at a reduced pressure of 60 Torr, a temperature of $800^{\circ} \mathrm{C}$, a hydrogen carrier gas flow of $50 \mathrm{slm}$, and a dichlorosilane $\left(\mathrm{SiCl}_{2} \mathrm{H}_{2}\right) \mathrm{Si}$ precursor flow of $296 \mathrm{sccm}$. The partial pressure of the As precursor gas arsine $\left(\mathrm{AsH}_{3}\right)$ was varied over a wide range and used as a free parameter to shape the As doping profile.

Typically in varactor applications, after the growth of a desired profile, a low-doped spacer layer is required at the surface. Figure 1 is a schematic of an $x^{-2}$ profile (region III) and a surface spacer layer (region II). To realize the abrupt drop in the doping, one must remove the As segregated on the surface almost completely, and no methods have been found to do this in the reactor. Therefore, the wafer is removed for an ex situ chemical cleaning in $\mathrm{HNO}_{3}$. Before further epitaxy, the surface must be hydrogen passivated by HF dipping. To preserve the already grown profile and prevent new As segregation from the already doped $\mathrm{Si}$, one must perform the following spacer epitaxy entirely at $800^{\circ} \mathrm{C}$. Therefore, after the HF dipping, we used Marangoni drying in an in-house fabricated system and epi pre-baking at $800^{\circ} \mathrm{C}$ to obtain the clean oxide-free surface necessary for achieving high-quality epitaxy.

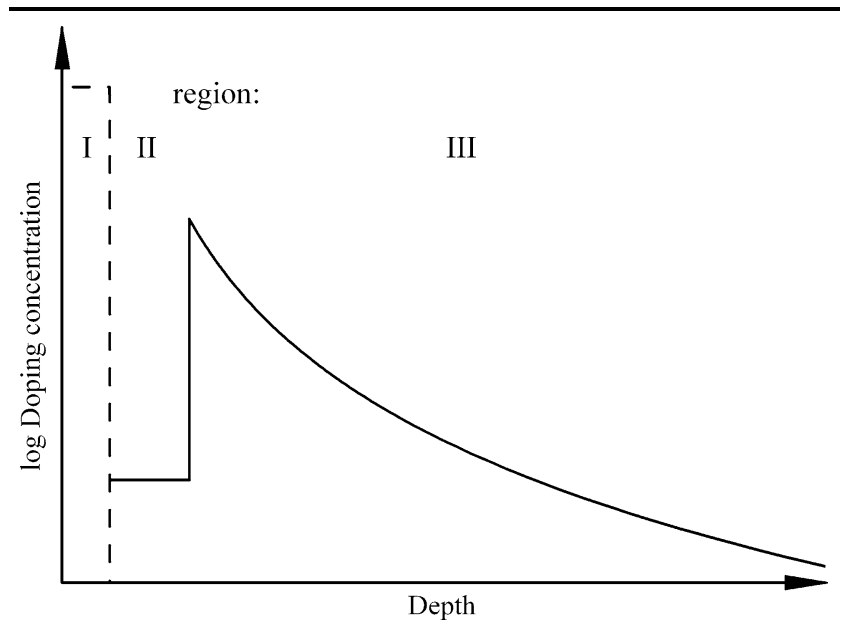

Fig. 1. Schematic of the desired $x^{-2}$ varactor doping profiles consisting of a lightly doped $n$-type surface layer (region II) on a region of ascending $n$-doping (region III), and an ultra-shallow $p^{+}$ surface layer (region I).
The profiles were integrated in $p^{+}-n$ diodes, where an ultra-shallow $p^{+}$region less than $5 \mathrm{~nm}$ deep was formed by deposition of pure B (region I in Fig. 1). ${ }^{14}$ This process is also performed in the Epsilon One reactor. Exposure to $\mathrm{B}_{2} \mathrm{H}_{6}$ causes boron atoms both to be adsorbed on the silicon surface in an amorphous phase and to react with $\mathrm{Si}$ in a boron-silicon phase. ${ }^{15}$ This is performed at $700^{\circ} \mathrm{C}$, so a limited diffusion of dopants into the Si ensures highly-doped ultra-shallow and ultra-abrupt junctions. The total B-deposition temperature can be kept as low as $700^{\circ} \mathrm{C}$, and no transient-enhanced diffusion is induced, so this step does not disturb the As doping profile in any way. The $p^{+}-n$ diodes were used for $C-V$ doping profiling of the As doped regions. In addition, the doping profiles were also extracted from a secondary ion mass spectroscopy (SIMS) analysis.

\section{ARSENIC SURFACE SEGREGATION MODEL AND PROFILE GROWTH}

In CVD epitaxial growth of $\mathrm{Si}$, As doping is achieved by the introduction of the arsine precursor gas during $\mathrm{Si}$ growth. At elevated temperatures arsine decomposes and $\mathrm{As}$ is adsorbed on the $\mathrm{Si}$ surface, but not immediately incorporated; instead, As continuously segregates to the growth surface. ${ }^{16}$ Only a small percentage of the As atoms will be incorporated, and, as a consequence, the doping is not proportional to the arsine partial pressure. This effect can be seen in Fig. 2a, where a profile grown with a constant arsine partial pressure is presented: the profile is not uniform but increases as the surface concentration of the As atoms increases. The increasing As surface concentration leads to saturation of the doping profile due to increased incorporation and decreased As adsorption.

The relationship between the As surface concentration and the doping level can be described by an incorporation rate $i_{R}$, a parameter proportional to the ratio of the doping obtained in the epitaxially grown layer and the surface coverage of As for which that doping is obtained. ${ }^{17} \mathrm{~A}$ dimensionless $i_{\mathrm{R}}$ is obtained by normalizing the doping to the $\mathrm{Si}$ concentration $N_{\mathrm{Si}}=5 \cdot 10^{22} \mathrm{~cm}^{-3}$, and defining the surface coverage $\theta$ as a percentage of the full monolayer coverage $\quad N_{\mathrm{ML}}=6.8 \cdot 10^{14} \mathrm{~cm}^{-2}{ }^{18}$ resulting in

$$
\frac{N_{\mathrm{d}}(x)}{N_{\mathrm{Si}}}=i_{\mathrm{R}} \theta(t)
$$

This has been used for the growth of slowly decreasing, nearly box-like, narrow, highly-doped profiles by first the deposition of the maximum possible amount of As on the Si surface and then the epitaxial growth of $\mathrm{Si}^{17}$

The purpose of our work was to extend the description of this mechanism to the case of continuous As doping during Si growth. Firstly, we 

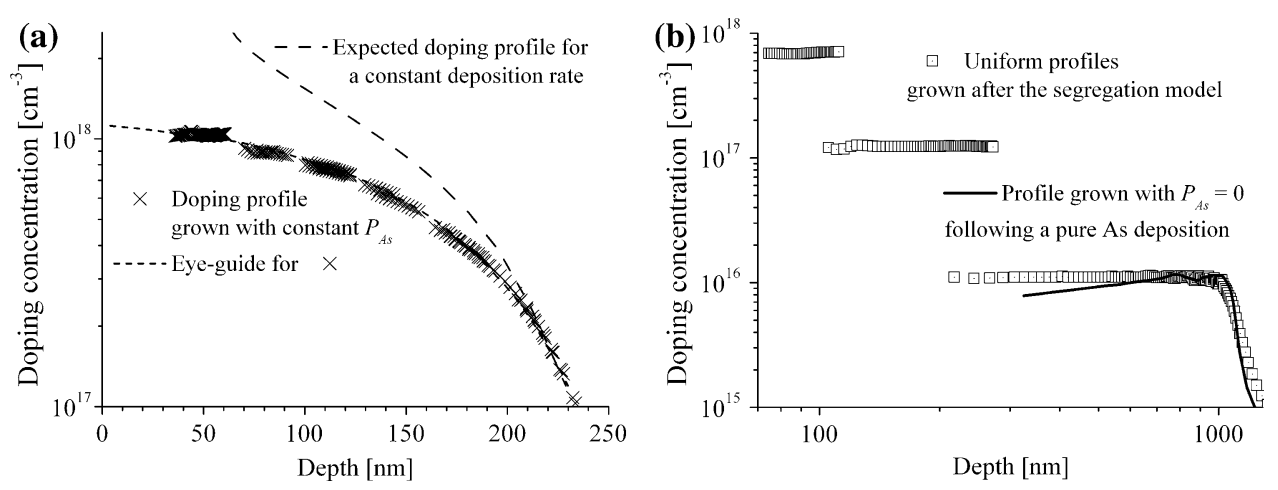

Fig. 2. Examples of different possible approaches to the growing of a uniform profile, measured by $C-V$. In (a), crosses represent the result of the application of constant arsine partial pressure during Si epitaxy, while in (b) the solid line is the profile obtained by As deposition followed by Si epitaxy, and the squares are the profiles achieved by both first As deposition and Si epitaxy with a constant arsine partial pressure, both tuned according to the presented model. As the profiles are obtained by $C-V$ measurements, only the section at depths covered by the depletion region boundary during reverse biasing from $0 \mathrm{~V}$ to the onset of high leakage or breakdown is presented.

made the assumption that the process could be described as a superimposed action of As adsorption and $\mathrm{Si}$ growth with $\mathrm{As}$ incorporation from the surface layer. Thereby, we describe the dynamics of this process by the growth rate $g_{R}$

$$
g_{\mathrm{R}}=\frac{\mathrm{d} x}{\mathrm{~d} t}
$$

and a continuity equation for the number of As atoms on the surface

$$
d\left(N_{\mathrm{ML}} \theta\right)=-N_{\mathrm{d}}(x) \mathrm{d} x+N_{\mathrm{ML}} d_{\mathrm{R}} P_{\mathrm{As}}(t) \mathrm{d} t
$$

where $d_{\mathrm{R}}$ is the As deposition rate. In this paper, As deposition strictly refers to the process of As adsorption as a consequence of wafer exposure to arsine at reduced pressure in the epitaxial reactor. It is reasonable to expect that the growth rate and the deposition rate will not be constant, as the sticking coefficients of $\mathrm{As}$ and $\mathrm{Si}$ depend on the surface As coverage, ${ }^{19}$ and, therefore, the second assumption that we made is contained in $g_{\mathrm{R}}=g_{\mathrm{R}}(\theta)$ and $d_{\mathrm{R}}=d_{\mathrm{R}}(\theta)$. The adsorption of As is self-limiting at $800^{\circ} \mathrm{C}$ and will, at most, yield a full monolayer coverage. ${ }^{10}$

The model proposed in Eqs. 1-3 relies on the values and dependencies of the three parameters: $i_{\mathrm{R}}, g_{\mathrm{R}}$, and $d_{\mathrm{R}}$. To test the assumptions of the model and to obtain these dependencies, we designed experiments to test each parameter as independently as possible from the others. The growth rate was obtained directly from the difference in wafer weight before and after epitaxial growth for samples with different As doping levels. Weight measurements yielded the thickness of the epitaxially grown layer with nanometer accuracy and relied on the uniformity of the epi layer, which had been previously confirmed by experiment. The incorporation rate was calculated from the doping decreasing slope after the arsine supply had been terminated, an example of which is given by the solid line in Fig. $2 b$, while the doping levels provided information about the As surface coverage and the deposition rate indirectly. For example, with the growth rate dependence obtained from the weight measurements, and the incorporation rate obtained from the decreasing slopes, if the deposition rate were constant, growth with a constant arsine partial pressure would be expected to follow the dashed line in Fig. 2a. From the difference between the dashed line and the measured data, information about the decrease in the deposition rate can be obtained. For the combination of the growth conditions used, as described in the previous section, the following approximate empirical dependencies were obtained:

$$
\begin{gathered}
g_{\mathrm{R}}=(0.72-2 \theta) \mathrm{nm} / \mathrm{s} \\
d_{\mathrm{R}}=(206-680 \theta) /(\text { Torr } \times \mathrm{s})
\end{gathered}
$$

These dependencies are linear approximations and give a good working relationship for the As surface coverage of up to $\theta \leq 10 \%$, but they can clearly not be used for $\theta$ approaching unity. The incorporation rate was found to be constant at

$$
i_{\mathrm{R}}=10^{-4}
$$

No dependency of any of the parameters on the arsine partial pressure was observed. It is known, however, that all the above parameters are temperature dependent: with decreasing temperature, the incorporation rate increases while the growth rate decreases, ${ }^{7}$ but the parameter space has not yet been explored in detail.

The successful extraction of dependencies given by Eqs. 4-6 confirms the assumption that the process can be regarded as a combined action of mutually independent As adsorption and Si growth with As incorporation, while the lack of dependence on the arsine partial pressure implies that there is no direct doping from the gaseous phase. From the dependencies obtained, a uniform profile, for example, can be grown by the combination of first a 
well-tuned pure As deposition and second Si growth with a well-tuned arsine partial pressure that would yield As deposition exactly equal to As incorporation. A few examples of such profiles are given in Fig. 2b.

This model makes it possible for one to fabricate a large variety of As doping profiles. Equations 1-6 form a system that can be numerically solved to give a time-dependent arsine partial pressure $P_{\mathrm{As}}(t)$ that would yield a desired doping profile $N_{\mathrm{d}}(x)$. Finally, the obtained time dependence of the arsine partial pressure must be discretized.

We developed a computer software tool that automatizes the numerical solution of the model equations, recipe discretization, recipe adjustments, if necessary, and, in addition, makes profile prediction based on the recipe possible. The last enables improvements to be made to the model if necessary, exploring the parameter space, and visualizing the consequences of recipe discretization.

\section{EXAMPLES OF EPITAXIALLY GROWN $x^{-2}$ PROFILES}

As an example of challenging profiles, we present here the results of the growth of a class of profiles proportional to the inverse square of the doping depth. These profiles were grown for novel varactor diodes with the purpose of distortion elimination. In order to serve this purpose successfully, the profiles must follow very accurately the mathematically given desired shape. Several $x^{-2}$ doping profiles were realized, two of which are discussed here. Figure 3 shows the accuracy of correspondence that we obtained between the mathematically optimal, intended, doping profiles, and the experimental doping profiles as measured either by SIMS (Fig. 3a) or $C-V$ profiling (Fig. 3b). Electrically measured profiles are preferred to those measured by SIMS for two reasons: first, the accuracy of the electrical measurement is better, and second, for varactor purposes, it is exactly the $C-V$ dependence that is important, and not the doping profile itself. However, as both SIMS and $C-V$ measurements result in profiles equal to the desired ones, as shown

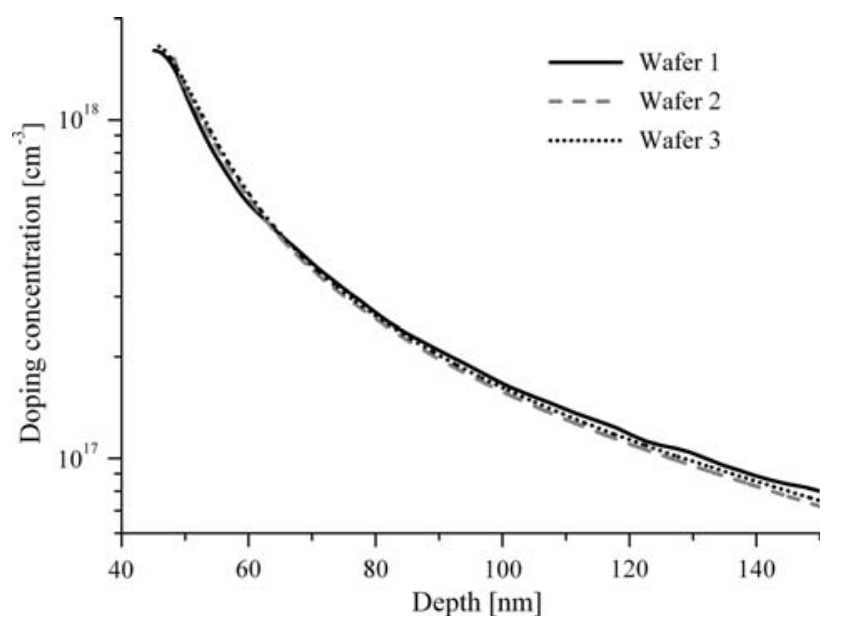

Fig. 4. The As doping profiles extracted from $C-V$ measurements on three different wafers that were processed with a time separation on the order of 1 month.

by Fig. 3 , it is also evident that the two measurement techniques reveal the same profiles. From this we can conclude that electrical activation is very close to $100 \%$, which is not surprising in the case of Si epitaxy. Figure 4 shows the excellent repeatability of the process. The profiles shown were fabricated independently with a time separation of approximately 1 month. However, the possibility that, over extended periods of time, a possible drift in the reactor temperature setting may lead to discrepancies that are more significant should not be excluded. Figure 5 shows the arsine partial pressure dependencies that were used in the reactor recipes to grow the profiles. The first step in the profile growth is pure As deposition, represented in Fig. 5 by a shaded box, while the zero on the time axis represents the onset of $\mathrm{Si}$ epi growth.

\section{THE RANGE OF ACHIEVABLE PROFILES}

The spectrum of doping profiles that can be grown is determined by the minimum and maximum achievable doping, and minimum and maximum achievable increasing and decreasing slopes, in the growth direction.
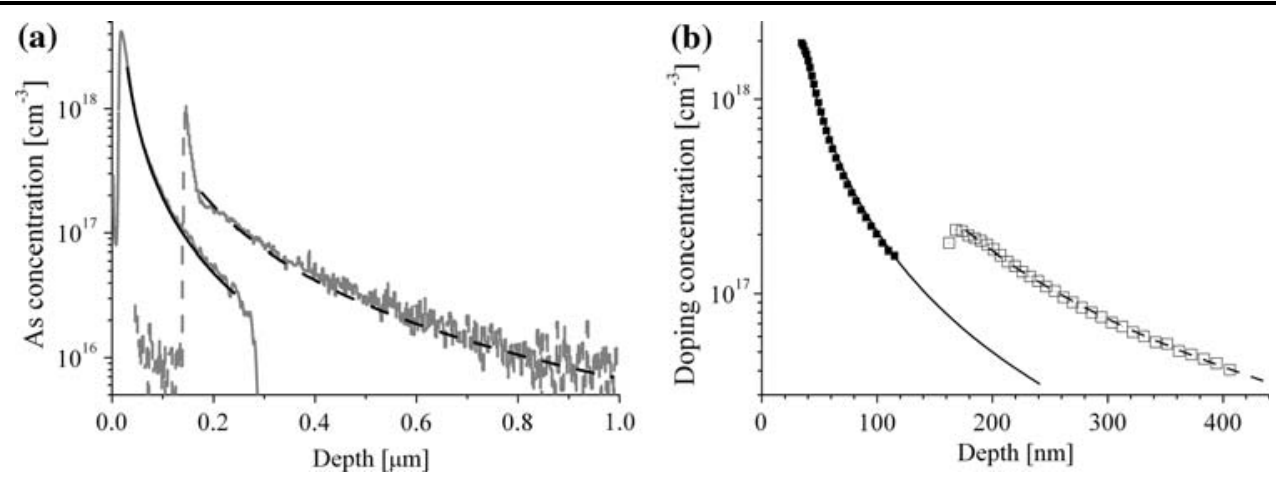

Fig. 3. Comparison of epitaxially grown As-doped $x^{-2}$ profiles measured by (a) SIMS (gray lines) and by (b) $C-V$ measurements (squares) to the desired theoretical profiles (black lines). 


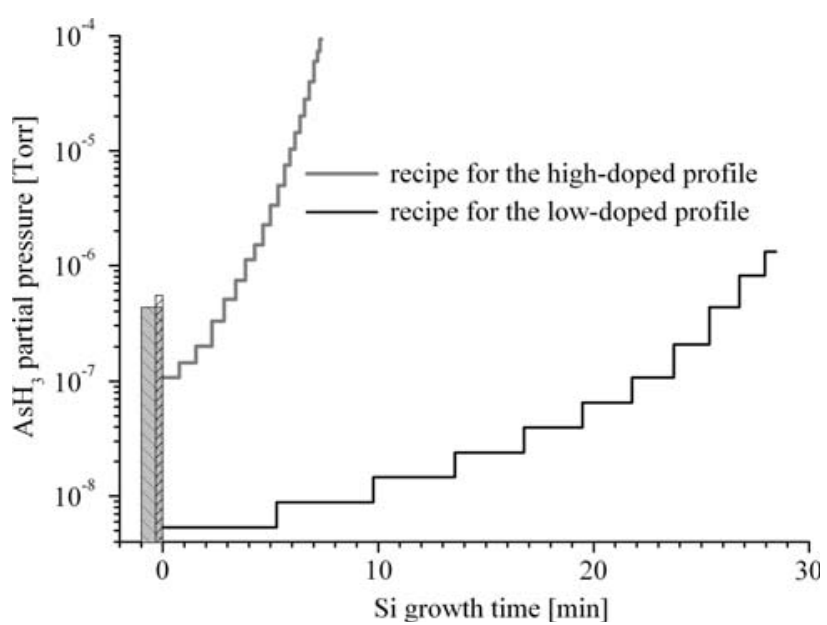

Fig. 5. Arsine partial pressure as a function of time in the recipes used for the epitaxial growth of the $x^{-2}$ region of the As doping profiles shown in Fig. 3. Zero on the time axis marks the end of pure As deposition and the start of simultaneous $\mathrm{Si}$ growth and As deposition.

No fundamental limit exists for the minimum achievable doping. It is determined by the minimum amount of arsine that can be supplied by the mass flow controllers in the reactor. In our setup this was approximately $10^{14} \mathrm{~cm}^{-3}$.

The maximum achievable doping is obtained with a full As monolayer coverage and is on the order of $5 \cdot 10^{18} \mathrm{~cm}^{-3}$ at $800^{\circ} \mathrm{C}$. It can be increased by either a reduction in the growth temperature ${ }^{7}$ or the introduction of, e.g., germanium as a catalyzer for incorporation. $^{17}$

The increasing slope range is determined by the rate at which the As surface coverage can increase during growth, which is determined by the arsine partial pressure. This limitation is, however, easily circumvented by the alternation of short periods of simultaneous As deposition and Si growth either with As deposition only or with $\mathrm{Si}$ growth only to effectively obtain faster or slower deposition, respectively.

Growth of a decreasing slope is related to the reduction of the As surface coverage. During growth, this is achieved by incorporation of As, but discontinuation of the arsine supply will yield only a slowly decreasing slope in the exponential form with the characteristic length given by $L=\frac{N_{\mathrm{ML}}}{i_{\mathrm{R}} N_{\mathrm{Si}}} \approx$ $1.36 \mu \mathrm{m}$, as shown by the solid line in Fig. 2b. For a more abrupt decrease of doping, surface segregated As can be completely removed outside the reactor, as specified in the "Experimental Procedures" section. ${ }^{20}$ The SIMS profiles in Fig. 3a demonstrate an abrupt drop in doping achieved in this manner. After As has been completely removed, the growth can be continued either with a clean As-free surface or from a certain doping level achieved by new, controlled, As deposition. This method is effective in reducing the doping level in the profile, but only in discrete steps. A smoothly decreasing profile would demand the controlled continuous removal of As from the surface during growth. In demanding applications, thermal desorption is not possible, due to diffusion-induced effects on the previously grown profile.

A small discrepancy may initially exist between the profile grown with the recipe obtained by the model presented and the desired doping profile. If the obtained accuracy is insufficient, the recipe used can be numerically scaled by multiplication of the arsine partial pressure in the recipe by the ratio of the desired and the measured profile slopes, rather than the doping levels, at the corresponding part of the profile. Most commonly, after the first scaling iteration a correspondence within measurement errors to the desired doping profile is obtained; however, e.g., the uniform profiles given in Fig. $2 b$ were grown directly, without scaling correction.

\section{CONCLUSIONS}

The developed model of As surface segregation during As-doped Si epitaxy describes the dynamics of As adsorption, segregation, and incorporation during the Si growth. It makes it possible for one to increase the surface segregated As in a controlled way and, thus, also As incorporation into the deposited $\mathrm{Si}$ layer. In this manner, arbitrarily shaped ascending doping profiles can be realized, as is described here, for specific $x^{-2}$ profiles. The segregated As is difficult to remove in situ, which prevents similar flexibility with respect to descending As profiles. Nevertheless, ex situ cleaning and Marangoni drying make the fabrication of abrupt drops in As doping possible.

\section{OPEN ACCESS}

This article is distributed under the terms of the Creative Commons Attribution Noncommercial License which permits any noncommercial use, distribution, and reproduction in any medium, provided the original author(s) and source are credited.

\section{REFERENCES}

1. W.D. van Noort, L.C.N. de Vreede, H.F.F. Jos, L.K. Nanver, and J.W. Slotboom, IEEE J. Solid State Circ. 36, 1399 (2001).

2. S. Yun and N. Guofu, Proceedings of IEEE Bipolar/ BiCMOS Circuits and Technology Meeting (2004), p. 213.

3. T. Sukegawa, T. Sakurai, and J. Nishizawa, IEEE Trans. Electron. Dev. 13, 988 (1966).

4. K. Lundien, R.J. Mattauch, J. Archer, and R. Malik, IEEE Trans. Microw. Theory 83, 235 (1983).

5. T.I. Kamins and D. Lefforge, J. Electrochem. Soc. 144, 674 (1997).

6. C.A. King, R.W. Johnson, T.Y. Chiu, J.M. Sung, and M.D. Morris, J. Electrochem. Soc. 142, 2430 (1995).

7. P.D. Agnello, T.O. Sedgwick, M.S. Goorsky, and J. Cotte, Appl. Phys. Lett. 60, 454 (1992).

8. S. Kinoshita, T. Konno, S. Takagi, T. Suzuki, and K. Maki, Jpn. J. Appl. Phys. 46, 5095 (2007). 
9. K.H. Jung, T.Y. Hsieh, D.L. Kwong, H.Y. Liu, and R. Brennan, Appl. Phys. Lett. 60, 724 (1992).

10. W.D. van Noort (Ph.D. Thesis, Delft University of Technology, Delft, 2001).

11. C. Huang, L.C.N. de Vreede, F. Sarubbi, M. Popadić, K. Buisman, J. Qureshi, M. Marchetti, A. Akhnoukh, T.L.M. Scholtes, and L.E. Larson, IEEE Trans. Microw. Theory 56, 1149 (2008).

12. L.K. Nanver, H. Schellevis, T.L.M. Scholtes, L.L. Spina, G. Lorito, F. Sarubbi, V. Gonda, M. Popadić, K. Buisman, L.C.N. de Vreede, C. Huang, S. Milosavljević, and E.J.G. Goudena, Proceedings of IEEE Bipolar/BiCMOS Circuits and Technology Meeting (2008), p. 33.

13. C. Huang, K. Buisman, L.K. Nanver, F. Sarubbi, M. Popadic, T.L.M. Scholtes, H. Schellevis, L.E. Larson, and L.C.N. de Vreede, IEEE Microw. Wirel. Compon. 18, 749 (2008).
14. F. Sarubbi, L.K. Nanver, and T.L. Scholtes, ECS Meeting Abstracts 602, 1000 (2006).

15. F. Sarubbi, L.K. Nanver, T. Scholtes, S.N. Nihtianov, and F. Scholze, Proceedings of the 38th European Solid-State Device Research Conference, ESSDERC (2008), pp. 278-281.

16. M.W.M. Graef, B.J.H. Leunissen, and H.H.C. de Moor, J. Electrochem. Soc. 132, 1942 (1985).

17. W.D. van Noort, L.K. Nanver, and J.W. Slotboom, J. Electrochem. Soc. 147, 4301 (2000).

18. A.K. Ott, S.M. Casey, and S.R. Leone, Surf. Sci. 405, 228 (1998).

19. H. Lüth, Surfaces and Interfaces of Solid Materials, 3rd ed. (Berlin: Springer, 1997).

20. M. Popadić, F. Sarubbi, T.L.M. Scholtes, S. Milosavljević, W. de Boer, and L.K. Nanver, Proceedings of Semiconductor Advances for Future Electronics (2006), pp. 475-478. 